\title{
Targeted treatment of metastatic castration-resistant prostate cancer with sipuleucel-T immunotherapy
}

\author{
Peter F. Mulders ${ }^{1} \cdot$ Maria De Santis $^{2} \cdot$ Thomas Powles $^{3} \cdot$ Karim Fizazi $^{4}$
}

Received: 3 December 2014 / Accepted: 29 April 2015 / Published online: 30 May 2015

(C) The Author(s) 2015. This article is published with open access at Springerlink.com

\begin{abstract}
Context Prostate cancer remains highly prevalent and has a poor clinical outcome once metastatic. Sipuleucel-T is an autologous cellular immunotherapy approved for the treatment of metastatic castration-resistant prostate cancer (mCRPC). Sipuleucel-T treatment extends survival but is independent of traditional short-term markers of treatment response observed with chemotherapy and contemporary hormonal treatments. Therefore, it is essential that clinicians understand the mechanism of action of sipuleucel-T and how this can translate in the clinic.

Objective This article aims to summarize the current knowledge of sipuleucel-T therapy and its effects in mCRPC.

Evidence acquisition Relevant publications describing sipuleucel-T clinical data and information relating to immunotherapies were identified.

Evidence synthesis Treatment with sipuleucel-T extends survival, with side effects being usually mild or moderate and manageable within the outpatient setting. The longterm immune responses generated by sipuleucel-T correlate with a survival benefit. Sipuleucel-T shows a greater

Peter F. Mulders

magnitude of clinical benefit when used in patients earlier in the mCRPC setting.

Conclusions Sipuleucel-T stimulates long-lived immune responses that translate into long-term clinical benefit. The treatment course (three infusions at weeks 0, 2, and 4) is associated with manageable side effects. Short-term markers of future benefit would be clinically useful, and information on effective treatment combinations or sequences is awaited.

Patient summary Sipuleucel-T treatment directs the patient's own immune system to target and remove prostate cancer cells and increases life expectancy. Patients whose cancer is less advanced generally have a more 'active' immune system and may benefit the most from this treatment.

Keywords Immunotherapy $\cdot$ Metastatic castrationresistant prostate cancer $\cdot$ Prostate cancer $\cdot$ Sipuleucel-T
Abbreviations
ADT Androgen deprivation therapy
APC Antigen-presenting cell
CD Cluster of differentiation
CI Confidence interval
CRPC Castration-resistant prostate cancer
DC Dendritic cell
DNA Deoxyribonucleic acid
EMA European Medicines Agency
FDA US Food and Drug Administration
GM-CSF Granulocyte-macrophage colony-stimulating factor
HR Hazard ratio
IFN $\gamma \quad$ Interferon gamma
IL Interleukin
LDH Lactate dehydrogenase

p.mulders@uro.umcn.nl

1 Department of Urology, Radboud University Nijmegen Medical Centre, P.O. Box 9101, 6500HB Nijmegen, The Netherlands

2 LBI-ACR VIEnna and 3rd Medical Department, KaiserFranz-Josef-Spital, Vienna, Austria

3 Barts Cancer Institute, Queen Mary University of London, London, UK
\end{abstract}




$\begin{array}{ll}\text { mCRPC } & \text { Metastatic castration-resistant prostate cancer } \\ \text { MHC } & \text { Major histocompatability complex } \\ \text { OS } & \text { Overall survival } \\ \text { PAP } & \text { Prostatic acid phosphatase } \\ \text { PBMC } & \text { Peripheral blood mononuclear cell } \\ \text { PD } & \text { Programmed cell death } \\ \text { PGWG2 } & \text { Prostate Cancer Working Group 2 } \\ \text { PFS } & \text { Progression-free survival } \\ \text { PSA } & \text { Prostate-specific antigen } \\ \text { QoL } & \text { Quality of life } \\ \text { SABR } & \text { Stereotactic ablative body radiation } \\ \text { TCR } & \text { T cell receptor } \\ \text { TNC } & \text { Total nucleated cell }\end{array}$

\section{Introduction}

Approximately 400,000 men are diagnosed with prostate cancer in Europe each year, equating to almost a quarter of all male cancers [1]. Around 90,000 prostate cancer-related deaths occur annually in Europe, giving it the third highest mortality rate after lung and colon cancers [1], and in the USA, it is surpassed only by lung cancer [2].

Men with localized prostate cancer are generally treated with active surveillance, brachytherapy, surgery, or radiotherapy with or without androgen deprivation therapy (ADT), depending on the individual prognosis [3]. Patients treated with ADT alone for metastatic disease usually respond well, but ultimately biochemical relapse and disease progression may occur. Castration-resistant prostate cancer (CRPC) may be suspected in patients with new symptoms, rising prostate-specific antigen (PSA) levels, or other new evidence of disease while being treated with ADT [4]. Treatment options for patients who progress to metastatic CRPC (mCRPC) have evolved dramatically over recent years and include the following: immunotherapy with sipuleucel-T [5, 6]; androgen biosynthesis inhibition with abiraterone acetate and prednisone [7]; androgen signaling inhibition with enzalutamide [8]; chemotherapy in combination with steroids, such as docetaxel or cabazitaxel and prednisone; and bone-targeted agents including the radioisotope radium-223 dichloride [9]. Additional immunotherapies are under investigation for $\mathrm{mCRPC}$, such as the cytotoxic T-lymphocyte antigen 4 inhibitor ipilimumab, for which phase III trial results were recently published [10].

Sipuleucel-T is an autologous cellular immunotherapy that is indicated for the treatment of asymptomatic or minimally symptomatic mCRPC [11]. In April 2010, sipuleucel-T became the first US Food and Drug Administration (FDA)-approved immunotherapy following the demonstration of significant overall survival (OS) improvement in patients with primarily asymptomatic or minimally symptomatic mCRPC $[5,12,13]$. Sipuleucel-T was also recently approved by the European Medicines Agency (EMA) for the treatment of asymptomatic or minimally symptomatic metastatic (non-visceral) CRPC in male adults in whom chemotherapy is not yet clinically indicated [14]. A European study of sipuleucel- $T$ is ongoing across centers in Vienna, Nijmegen, London, and Paris, with the product manufactured in Maastricht (The Netherlands) using the standard methodology (see section 'Manufacture of sipuleucel-T'). In addition, sipuleucel-T is being explored in earlier and later settings, and the initial findings have been positive [15]. In clinical trials, sipuleucel-T was well tolerated and had manageable side effects [16]; the majority of adverse events were mild or moderate and could be managed in an outpatient setting. In order to appreciate the long-term clinical benefits of sipuleucel-T treatment, it is important to understand how these are derived from its mode of action.

\section{Evidence acquisition}

Relevant publications presenting data on sipuleucel-T treatment, predominantly from key clinical trials, were identified. Additional articles commenting on these data, and on the results achieved with sipuleucel-T and other immunotherapies, were also included.

\section{Evidence synthesis}

Data and other information from the identified publications were collated and are presented below.

\section{Manufacture of sipuleucel-T}

Sipuleucel-T uses the patient's own cells to stimulate an immune response against prostatic acid phosphatase (PAP), which is an appropriate target as it is expressed almost exclusively by prostate cells. The potential for inappropriate immune reactions is limited because sipuleucel- $T$ is composed of the patient's own cells.

The first step in the manufacture of sipuleucel-T is to isolate the patient's peripheral blood mononuclear cells (PBMCs), including antigen-presenting cells (APCs) such as dendritic cells, macrophages, and B cells, using leukapheresis (Fig. 1). These cells are then cultured in vitro for $36-44 \mathrm{~h}$ with a fusion protein, PA2024, composed of recombinant PAP fused to granulocyte-macrophage colony-stimulating factor (GMCSF), a cytokine that stimulates APCs. Stimulating APCs ex vivo is thought to be an effective approach for initiating an immune response away from the potentially immunosuppressive effects of the prostate tumor. Ex vivo PA2024-activated APCs have been shown to process and display fragments 


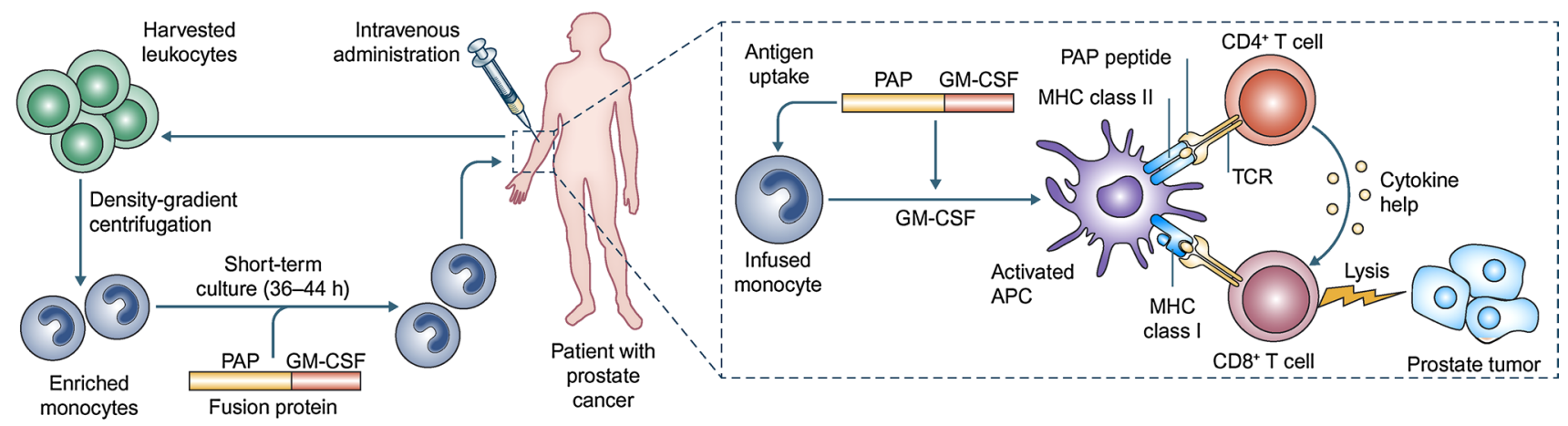

Fig. 1 Sipuleucel-T immunotherapy and proposed mode of action. Leukocytes are harvested from the patient by leukapheresis and sent for processing, in which monocytes are enriched by density-gradient centrifugation. The monocytes are incubated with the PA2024 fusion protein of PAP and GM-CSF. PA2024 is taken up by immature APCs, such as DCs, and enhances their maturation. The resulting 'product' is returned to the clinic and administered intravenously to the patient. Theoretically, the transfused APCs will present PAP-derived peptides to the host immune system in vivo, activating $\mathrm{CD} 4^{+}$and $\mathrm{CD} 8^{+} \mathrm{T}$

of the PAP moiety on the cell surface [18]. The sipuleucel$\mathrm{T}$ product is then reinfused back into the patient, where the activated APCs stimulate an immune response against PAP and consequently against the cancerous prostatic cells. The potency of sipuleucel-T may be assessed by the upregulation of cluster of differentiation 54 (CD54) on APCs (measured as an increase in the ratio of the average number of molecules of CD54 on APCs pre- vs post-culture with PA2024) [18]. Cumulative APC activation and APC number correlated with OS in an analysis of three randomized controlled phase III trials [19]. Thus, current sipuleucel-T manufacture requires that the product contains a minimum of 50 million autologous $\mathrm{CD} 54^{+}$cells [11]. The complete treatment involves three cycles 2 weeks apart (weeks 0,2 , and 4), with each cycle comprising fresh leukapheresis, cell isolation and culture with PA2024, and reinfusion. The sipuleucel- $\mathrm{T}$ product contains $\mathrm{T}$ cells, APCs, natural killer cells, and B cells, and the cellular composition does not change between treatment weeks nor between pre-culture compared with the final product [19].

\section{Engagement of the immune system during manufacture of sipuleucel-T}

Significant levels of in vitro APC activation, measured by CD54 upregulation, are induced during co-culture of the patient's cells with PA2024 [18-20]. Greater increases in APC activation are seen during the second and third (weeks 2 and 4 ) in vitro co-cultures compared with the first (week $0)$. This response profile is akin to that generated by traditional vaccines-a prime-boost effect is observed after the first dose when administering sequential vaccinations. Significant positive correlations were observed between OS cells and initiating adaptive immune responses. Adapted by permission from Macmillan Publishers Ltd: Nature Reviews Immunology 2010, Vol. 10, Drake CG, Prostate cancer as a model for tumor immunotherapy, pp. 580-93, copyright 2010 [17]. APC antigen-presenting cell. $C D$ cluster of differentiation. $D C$ dendritic cell. GM-CSF granulocyte-macrophage colony-stimulating factor. $M H C$ major histocompatability complex. PA2024 fusion protein of PAP and GM-CSF. PAP prostatic acid phosphatase. TCR T cell receptor

and cumulative (across the three infusions) post-culture in vitro APC activation, APC count, and total nucleated cell (TNC) count, which remained after adjusting for baseline PSA and lactate dehydrogenase (LDH) levels (Fig. 2) [19].

There is evidence to suggest that antigen-specific $\mathrm{T}$ and $\mathrm{B}$ cell responses developed following the first sipuleucel-T infusion, and that these were re-stimulated during the subsequent co-cultures. Cytokines that are associated with $\mathrm{T}$ cell activation were detected in culture medium from the second and third in vitro co-cultures, and included interleukin (IL)-2, IL-4, IL-5, IL-6, IL-10, IL-13, IL-17, interferon gamma (IFN $\gamma$ ), IFN $\gamma$ inducible protein 10, and tumor necrosis factor alpha [19, 20]. In addition, levels of the $\mathrm{T}$ cell activation markers CD134 and $\mathrm{CD} 137$ were increased on $\mathrm{CD}^{+}{ }^{+}$and $\mathrm{CD}^{+}{ }^{+} \mathrm{T}$ cells after culture with PA2024, and there was evidence of B cell maturation and activation [20]. Furthermore, PA2024-specific T cell 'recall' responses, measured by antigen-specific proliferation and IFN $\gamma$ production, could be detected in pre-culture cells from the second and particularly the third leukapheresis [19, 20]. This suggests that functional $\mathrm{T}$ cell responses were generated in the body after the first infusion and were subsequently boosted after the second infusion. Each of these findings reinforces the hypothesis that sipuleucel-T treatment induces an immunologic 'prime-boost' response.

\section{Post-treatment immune responses with sipuleucel-T and correlation with $\mathrm{OS}$}

$\mathrm{T}$ cell responses, such as proliferation and IFN $\gamma$ production in response to stimulation with either PA2024 or PAP, could be detected in PBMCs from the majority of patients treated with sipuleucel-T [19]. This indicates that the treatment 

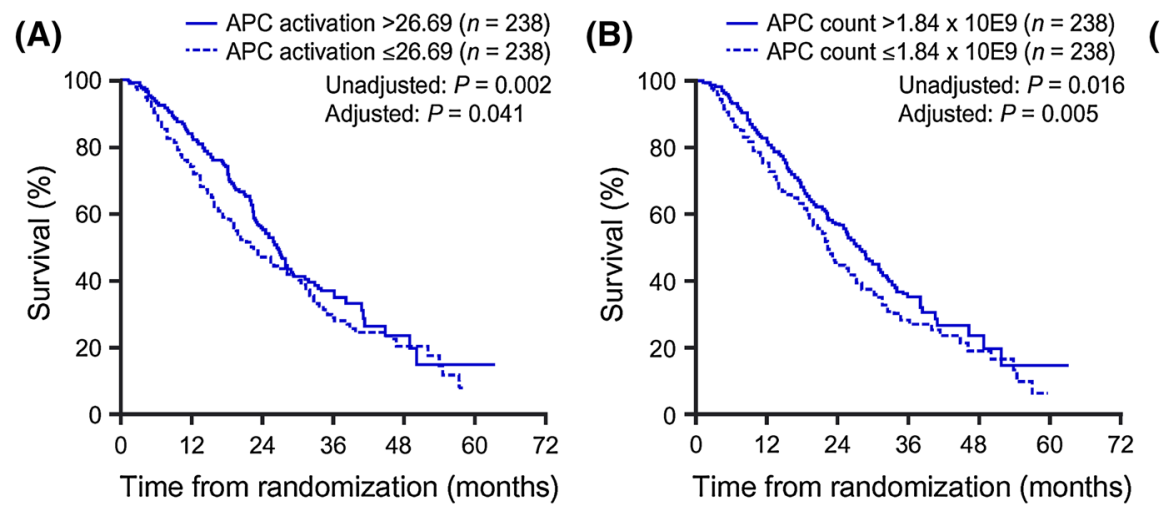

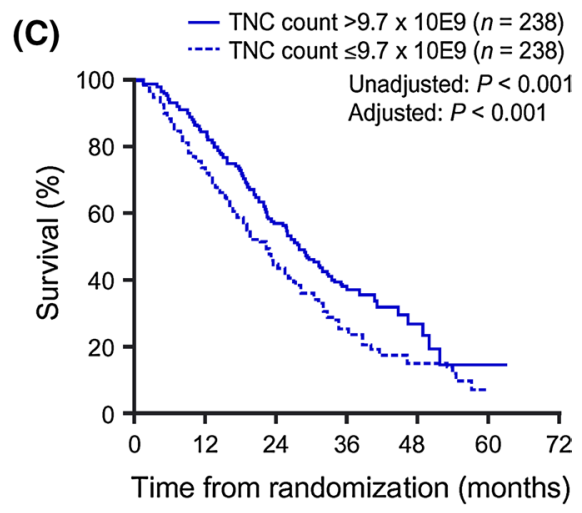

Fig. 2 Significant correlation between OS and cumulative a APC activation, b APC $\left(\mathrm{CD} 54^{+}\right.$cell) count, and $\mathbf{c}$ TNC count (product parameters) following co-culture of cells with PA2024. KaplanMeier survival plots are shown for each product parameter above versus below the median value; $p$ values from analysis of each parameter as a continuous measure were calculated with and without adjustment for baseline PSA and LDH levels. Reproduced from Cancer Immunology Immunotherapy 2013, Vol. 62, Sheikh NA, Petrylak D,

generated prostate antigen-specific immune responses. Preliminary evidence also suggests that this peripheral blood immune response has a direct effect within the prostate, as demonstrated by an increased number of leukocytes around the tumor site [21]. In the neoadjuvant setting, patients with non-metastatic prostate cancer who were treated with sipuleucel-T displayed $\mathrm{a} \geq 3$-fold increase in mean cells/area at the tumor interface for total $\mathrm{T}$ cells, $\mathrm{CD} 4^{+}$helper $\mathrm{T}$ cells, and $\mathrm{CD}^{+}$cytotoxic $\mathrm{T}$ cells, compared with pre-treatment biopsy or with non-interface areas of benign or malignant tissue [21].

Robust post-treatment antibody responses specific to PA2024 and PAP have also been detected in the majority of patients treated with sipuleucel-T, but not in control patients [19]. In the majority of sipuleucel-T-treated patients, antigen-specific antibodies remained present at detectable levels in serum for at least 26 weeks. The majority of the antibody responses were initially immunoglobulin $\mathrm{M}$, with immunoglobulin $\mathrm{G}$ antibodies emerging 2-4 weeks after the last infusion. This isotype switching is consistent with vaccine-induced immunologic memory. In addition, anti-tetanus antibody responses were unchanged after sipuleucel-T treatment, indicating that bystander immune responses were not generated.

Antibodies in the systemic circulation may, theoretically, reach many tissues, potentially allowing the destruction of malignant cells throughout the body and resulting in long-term, widespread immunosurveillance. There is also potential for the immune system to recognize additional 'nontarget' cancer antigens through a process known as epitope spreading, to enable it to better identify and remove cancerous cells. Epitope spreading is particularly noted in the development of long-term antibody responses. This antigen cascade event may explain why OS
Kantoff PW, et al., Sipuleucel-T immune parameters correlate with survival: an analysis of the randomized phase 3 clinical trials in men with castration-resistant prostate cancer, pp. 137-47. Copyright 2012, the Author(s) [19]. APC antigen-presenting cell, $C D$ cluster of differentiation, $G M-C S F$ granulocyte-macrophage colony-stimulating factor, $L D H$ lactate dehydrogenase, $O S$ overall survival, PA2024 fusion protein of PAP and GM-CSF, PAP prostatic acid phosphatase, PSA prostate-specific antigen, $T N C$ total nucleated cell

significantly correlated with an antibody response against PA2024 [hazard ratio (HR) 0.42; $p<0.001$ ] [19] and highlights the importance of humoral immunity in this context. OS also significantly correlated with the development of either a functional $\mathrm{T}$ cell response (above) or a humoral response (Fig. 3).

Overall, these data support the concept that engagement of key immune cell types (APCs, T, and B cells) during sipuleucel$\mathrm{T}$ treatment contributes to the OS benefit. However, there are additional data to suggest that engagement of 'less prominent' immune cell types could also be important. Approximately $30 \%$ of the patients in three phase III trials experienced a transient increase in peripheral blood eosinophil counts from week 6 to week 14, directly after completion of sipuleucel-T treatment [22]. Eosinophils are often considered to be rare, cytotoxic cells that are only relevant in parasitic infections and asthma [23]. However, there is increasing evidence that eosinophils may be involved in the generation of sipuleucel-T antitumor immune responses [23]. This may be through interactions with $\mathrm{T}$ cells (recruitment and polarization or direct antitumor activity), B cells (antibody responses), or dendritic cells (activation/recruitment). Indeed, the transient, elevated eosinophil counts in patients receiving sipuleucel-T correlated with OS, prostate cancer-specific survival, and antigen-specific humoral responses [22].

\section{Optimizing the use of sipuleucel-T}

\section{Surrogate markers for OS}

Unlike traditional chemotherapy, sipuleucel-T treatment is associated with a long-term OS benefit but not a reduction in time to objective disease progression (defined by at least one of the following criteria: an increase in at least $50 \%$ in 

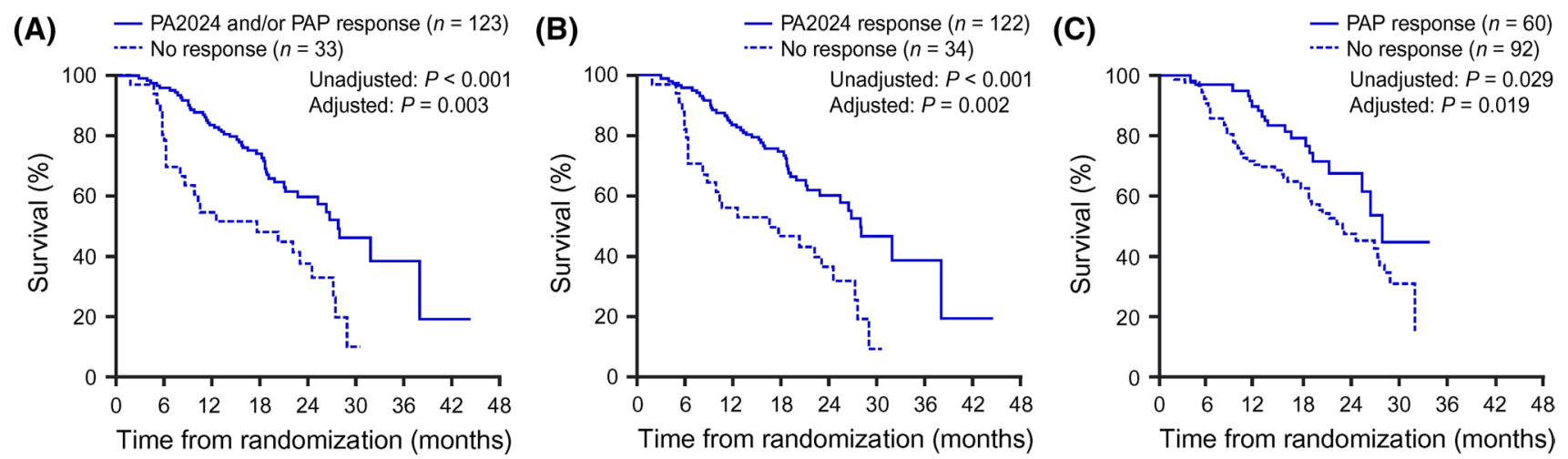

Fig. 3 Significant correlation between OS and immune responders or non-responders to sipuleucel-T treatment as determined by a response to either PA2024 or PAP, b response to PA2024, and c response to PAP, in at least one of the three immune response assays (antibodies, IFN $\gamma$-production, and proliferation). Kaplan-Meier survival plots are shown; $p$ values from analysis of each parameter were calculated with and without adjustment for baseline PSA and LDH levels. Reproduced from Cancer Immunology Immunotherapy 2013,

the sum of the products of diameters for index lesions; the new appearance or unequivocal progression of non-index lesions; at least two new lesions on bone scanning; and/or a new pathologic fracture or spinal cord compression [5]). The same phenomenon has been noted with other immunotherapies. For example, treatment with PSA-TRICOM did not increase time to prostate cancer progression but achieved a significant improvement in 3-year OS $\{30 \%$ vs. $17 \%$; HR 0.56 [95 \% confidence interval (CI), 0.37-0.85] \} and median survival (25.1 vs. 16.6 months; $p=0.0061$ ) versus control [6]. Similarly, in melanoma, ipilimumab treatment did not result in an improvement in median progression-free survival (PFS), but a significant extension of median OS was achieved (10.1 months in ipilimumab arms) compared with active control (6.4 months; $p<0.001$ and $p=0.003$, respectively) [24]. Therefore, traditional shortterm indicators of long-term survival benefits may not be appropriate with sipuleucel-T or other immunotherapies.

This seeming discrepancy may relate to the immunomodulatory mode of action. Antigen-specific immune responses take time to generate, and therefore tumor shrinkage may be delayed compared with traditional therapies (Fig. 4a) [25, 26]. It is also likely that an initial inflammatory influx of leukocytes into a tumor could lead to an initial, nonpathogenic increase in size, followed by a subsequent, long-term antitumor response [25]. Thus, immunologic memory could enable the body to continue to fight the tumor and slow its growth long after the immunotherapeutic treatment has been completed [25, 26]. Furthermore, theoretically, long-term immunosurveillance could act throughout the body as well as within the prostate, and could potentially target metastatic cells, limiting their spread.
Vol. 62, Sheikh NA, Petrylak D, Kantoff PW, et al., Sipuleucel-T immune parameters correlate with survival: an analysis of the randomized phase 3 clinical trials in men with castration-resistant prostate cancer, pp. 137-47. Copyright 2012, the Author(s) [19]. GM-CSF granulocyte-macrophage colony-stimulating factor. $I F N \gamma$ interferon gamma. $L D H$ lactate dehydrogenase. $O S$ overall survival. PA2024 fusion protein of PAP and GM-CSF. PAP prostatic acid phosphatase. $P S A$ prostate-specific antigen
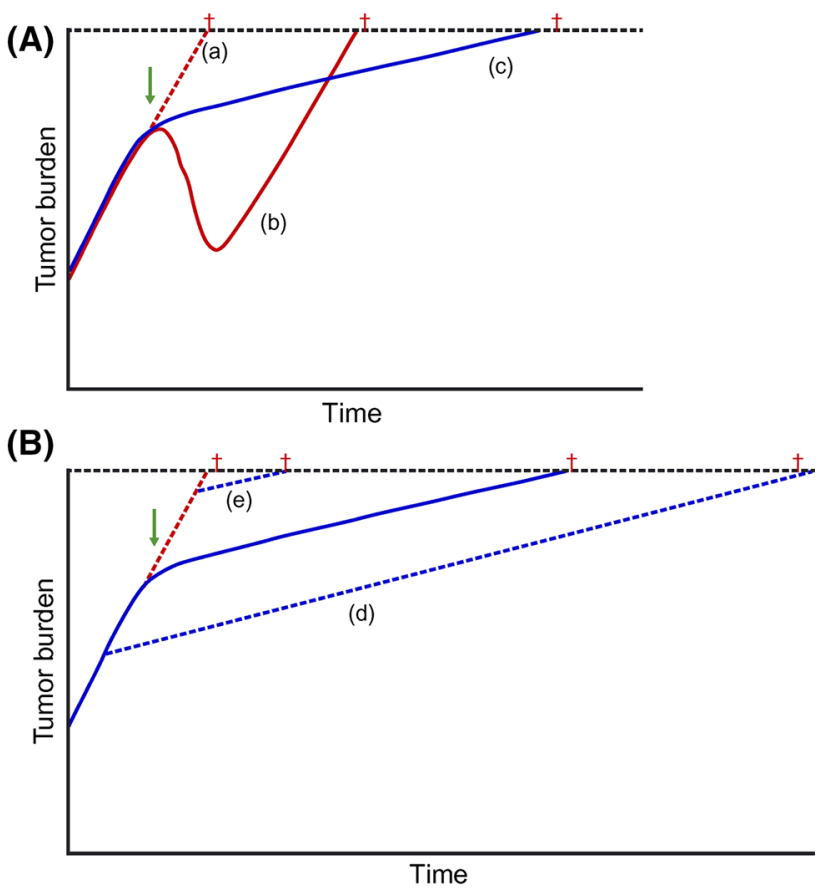

Fig. 4 Proposed kinetics of immunomodulatory treatments. In a, immunotherapy is compared with cytotoxic chemotherapy. Tumor burden is shown if $a$ no therapy is initiated, $b$ chemotherapy is initiated, or $c$ immunomodulatory therapy is initiated. For patients who received immunotherapy, there may be little if any reduction in tumor size, and therefore little or no increase in time to progression, but an increase in OS. Dagger denotes time of death. In b, early versus late initiation of immunomodulatory therapy is explored. The survival benefit may be increased if immunomodulatory treatment is initiated earlier in disease progression $(d)$ but may be decreased in patients with a large tumor burden (e). Reproduced from Schlom J. Therapeutic cancer vaccines: current status and moving forward. J Natl Cancer Inst 2012;104(8):599-613, by permission of Oxford University Press [31]. OS overall survival 


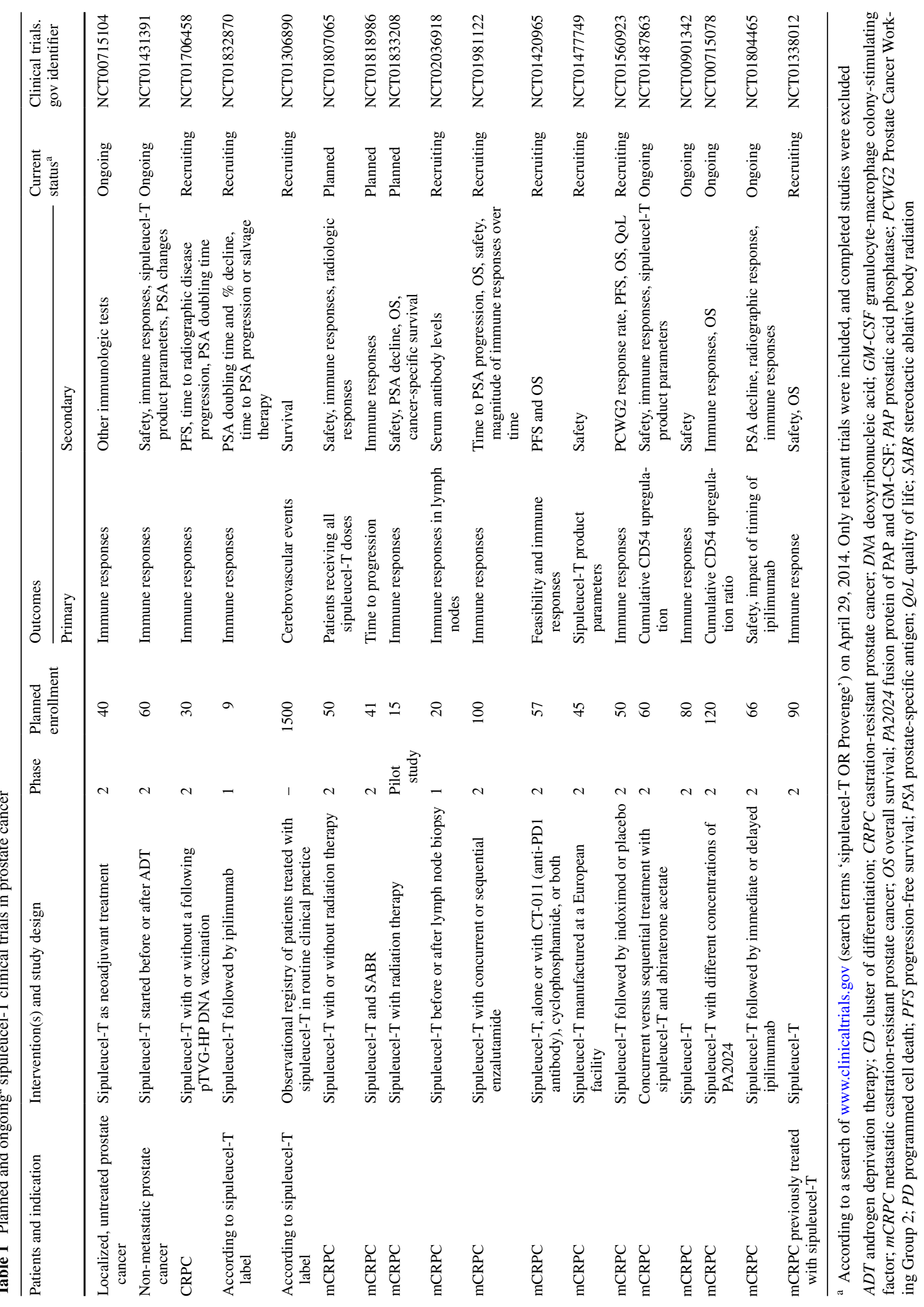


Clinically, short-term markers of future benefit from immunotherapies would be very useful. In this regard, the transient increase in eosinophil counts in peripheral blood, noted from week 6 to week 14, may play a role given that this correlates with OS [22]. Eosinophil counts are routinely measured in this context; therefore, after further investigation and validation, elevated eosinophil counts could be a potentially useful surrogate marker.

\section{Sipuleucel-T's position within the treatment paradigm}

Sipuleucel-T was evaluated in three randomized phase III studies [D9901: NCT00005947; D9902A: NCT01133704; and Immunotherapy for Prostate Adenocarcinoma Treatment (IMPACT; D9902B): NCT00065442] of patients with asymptomatic or minimally symptomatic mCRPC, and consistent improvements in OS, compared with placebo, were observed across the studies [5, 12, 13, 16]. FDA and EMA approval of sipuleucel-T was based primarily on the pivotal IMPACT study which demonstrated that sipuleucel-T was associated with improved median survival (4.1 months; 25.8 vs. 21.7 months in the sipuleucel-T and control arms, respectively), increased 3-year survival (31.7 vs. $23.0 \%$ in the sipuleucel-T and control arms, respectively) and a $22.5 \%$ reduction in risk of death (HR 0.78; $95 \%$ CI $0.61-0.98 ; p=0.03)$. Although there were more adverse events in the sipuleucel-T group, compared with the placebo group, these events were primarily related to leukapheresis and infusion. Most events were mild to moderate (typically chills, fever, fatigue, nausea, and/or headache), occurred within 1 day of infusion, and resolved within 1-2 days, with very few patients $(0.9 \%)$ prematurely discontinuing sipuleucel-T therapy [5].

A commentary by Huber et al. [27] questioned the trial design and the validity of the results from the IMPACT study. The following concerns were raised: (1) unexpected interactions between patient age and survival, (2) older patients in the placebo group appeared to have shorter OS than might be expected based on the results of other studies, and (3) the number of cells harvested and reinfused to the patients. Three subsequent publications rebutted these concerns [28-30] and presented clear explanations as to why, due to comparisons between asymmetric and small subgroups, and a lack of evaluable data, the article by Huber et al. was misleading. Moreover, independent bodies, such as the FDA and the Center for Medicare and Medicaid Services, also found the issues raised by Huber et al. not to be credible, supporting the published rebuttals. In summary, the claims made by Huber et al. were considered to be unfounded, a view supported overwhelmingly by the regulatory bodies and key thought leaders in the field.

Further to the above-summarized clinical trial data supporting improvements in OS of patients with asymptomatic or minimally symptomatic mCRPC treated with sipuleucel$\mathrm{T}$, several lines of evidence support sipuleucel- $\mathrm{T}$ use earlier within the treatment paradigm. Treating patients earlier in the disease course would allow more time for the immune response to develop and would theoretically allow patients to benefit most from the long-term OS advantage (Fig. 4b). In addition, several factors associated with improved survival following sipuleucel-T treatment, such as APC activation and transient elevations in eosinophil counts, are greater or more frequent in patients with characteristics of earlier-stage disease $[15,22,27]$. Indeed, the cumulative fold increase in APC activation, as measured by CD54 upregulation, was significantly greater in patients with earlier-stage disease; the fold increase was 35.5 in neoadjuvant patients, 28.7 in asymptomatic or minimally symptomatic mCRPC patients, and 21.8 in mCRPC patients $(p<0.0001$; Joncheere-Terpstra test) [31]. In addition, patients with characteristics of earlier-stage disease may show greater OS [32], a greater time to first opioid analgesic use, and a trend toward a delay in time to disease-related pain after sipuleucel-T treatment than those with later-stage disease characteristics [33].

\section{Future directions with sipuleucel-T in the management of prostate cancer}

Several ongoing and planned studies will investigate further the optimal role for sipuleucel-T in the management of prostate cancer (Table 1). These trials are addressing several aspects of sipuleucel-T therapy, such as use in earlier treatment settings (neoadjuvant, hormone sensitive, and non-metastatic) and the combination of sipuleucel-T with other agents (chemotherapy, radiotherapy, ADT, abiraterone acetate, and immunotherapeutics). Use of sipuleucel-T earlier in the prostate cancer paradigm may have considerable benefits to patients, since these patients are generally able to mount more robust immune responses [15, 22, 27], and there may be more time for the sustained, durable immune responses to exert their clinical benefit [32-34]. Preliminary data on the use of sipuleucel-T before, concurrently with, or after additional anticancer therapies are also encouraging. In the IMPACT trial, sipuleucel-T use was associated with increased survival versus placebo irrespective of previous chemotherapy or docetaxel use, radiotherapy, prostatectomy, orchiectomy, castration, or combined androgen blockade [5]. In addition, a sipuleucel-T treatment effect was observed in patients with and without subsequent docetaxel use [35]. Preliminary data from the P11-3 study suggest that treatment with abiraterone acetate and prednisone does not substantially affect the leukocyte yield for most patients, and that sipuleucel-T can, therefore, be successfully manufactured alongside the use of these agents [36]. The potential effects of treatment sequencing on clinical outcomes are also under investigation. Preliminary interim results 
from the phase II STAND trial (sequencing of sipuleucel$\mathrm{T}$ and ADT in men with non-metastatic prostate cancer; NCT01431391) show that combination treatment with ADT followed by sipuleucel-T may augment immune responses, which could potentially increase efficacy compared with either treatment alone [37].

\section{Conclusions}

New, effective drugs with different mechanisms of action are essential for the treatment of prostate cancer, which remains highly prevalent and has both a high mortality rate and poor clinical outcome after biochemical relapse. Sipuleucel-T is a significant advance in the treatment of mCRPC and provides clinicians with an additional effective therapy. Treatment with sipuleucel-T extends survival and is associated with easily manageable side effects, which are usually mild. Few patients prematurely discontinue sipuleucel-T therapy, and substantial long-term benefits can be achieved with just a 4-week treatment course. Research is ongoing to identify short-term markers of the long-term clinical benefits of sipuleucel-T treatment, to investigate its use in earlier disease settings, and in combination with other therapies.

Acknowledgments Medical writing and coordination assistance was provided by Stuart Langley and Joanne Swainston of GardinerCaldwell Communications and funded by Dendreon Corporation.

Conflict of interest Peter F. Mulders is a speaker and participates in clinical trials for Dendreon Corporation. Maria De Santis has received consultancy fees from Amgen, Astellas, Bayer, Celgene, Dendreon Corporation, Ferring, GSK, Ipsen, Janssen Cilag, Novartis, Pfizer, Pierre Fabre Oncology, Roche, Sanofi, Shionogi, and Teva/OncoGenex. Thomas Powles has received honoraria from Dendreon Corporation. Karim Fizazi is an advisory board participator and speaker for Dendreon Corporation.

Ethical standards The manuscript does not contain original clinical study or patient data, all data has previously been published and appropriately referenced herein.

Open Access This article is distributed under the terms of the Creative Commons Attribution 4.0 International License (http://creativecommons.org/licenses/by/4.0/), which permits unrestricted use, distribution, and reproduction in any medium, provided you give appropriate credit to the original author(s) and the source, provide a link to the Creative Commons license, and indicate if changes were made.

\section{References}

1. Ferlay J, Steliarova-Foucher E, Lortet-Tieulent J et al (2013) Cancer incidence and mortality patterns in Europe: estimates for 40 countries in 2012. Eur J Cancer 49:1374-1403

2. Siegel R, Ma J, Zou Z, Jemal A (2014) Cancer statistics, 2014. CA Cancer J Clin 64:9-29
3. Horwich A, Hugosson J, de Reijke T, Panel members: European Society for Medical Oncology et al (2013) Prostate cancer: ESMO consensus conference guidelines 2012. Ann Oncol 24(Suppl 5):1141-1162

4. Hotte SJ, Saad F (2010) Current management of castrate-resistant prostate cancer. Curr Oncol 17(Suppl 2):S72-S79

5. Kantoff PW, Higano CS, Shore ND et al (2010) Sipuleucel-T immunotherapy for castration-resistant prostate cancer. N Engl J Med 363:411-422

6. Kantoff PW, Schuetz TJ, Blumenstein BA et al (2010) Overall survival analysis of a phase II randomized controlled trial of a Poxviral-based PSA-targeted immunotherapy in metastatic castration-resistant prostate cancer. J Clin Oncol 28:1099-1105

7. Ryan CJ, Smith MR, de Bono JS et al (2013) Abiraterone in metastatic prostate cancer without previous chemotherapy. N Engl J Med 368:138-148

8. Scher HI, Fizazi K, Saad F et al (2012) Increased survival with enzalutamide in prostate cancer after chemotherapy. N Engl J Med 367:1187-1197

9. Parker C, Nilsson S, Heinrich D et al (2013) Alpha emitter radium-223 and survival in metastatic prostate cancer. $\mathrm{N}$ Engl $\mathrm{J}$ Med 369:213-223

10. Kwon ED, Drake CG, Scher HI et al (2014) Ipilimumab versus placebo after radiotherapy in patients with metastatic castrationresistant prostate cancer that had progressed after docetaxel chemotherapy (CA184-043): a multicentre, randomised, doubleblind, phase 3 trial. Lancet Oncol 15:700-712

11. Dendreon Corporation. PROVENGE (sipuleucel-T) prescribing information. http://www.dendreon.com/prescribing-information. pdf. Accessed 27 Oct 2014

12. Small EJ, Schellhammer PF, Higano CS et al (2006) Placebocontrolled phase III trial of immunologic therapy with sipuleucel-T (APC8015) in patients with metastatic, asymptomatic hormone refractory prostate cancer. J Clin Oncol 24:3089-3094

13. Higano CS, Schellhammer PF, Small EJ et al (2009) Integrated data from two randomized, double-blind, placebo-controlled, phase 3 trials of active cellular immunotherapy with sipuleucel-T in advanced prostate cancer. Cancer 115:3670-3679

14. Dendreon UK Limited. PROVENGE ${ }^{\circledR}$ (sipuleucel-T) summary of product characteristics. http://www.ema.europa.eu/ docs/en_GB/document_library/EPAR_-_Product_Information/ human/002513/WC500151099.pdf. Accessed 27 Oct 2014

15. Small EJ, Wesley JD, Quinn D et al (2012) Antigen-presenting cell (APC) activation in sipuleucel-T: is activation increased in early prostate cancer disease states? European Society for Medical Oncology (ESMO) Congress, Vienna, Austria, September 28-October 2, 2012. Poster No. 942

16. Hall SJ, Klotz L, Pantuck AJ et al (2011) Integrated safety data from four randomized, double-blind, controlled trials of autologous cellular immunotherapy with sipuleucel-T in patients with prostate cancer. J Urol 186:877-881

17. Drake CG (2010) Prostate cancer as a model for tumour immunotherapy. Nat Rev Immunol 10:580-593

18. Sheikh NA, Jones LA (2008) CD54 is a surrogate marker of antigen presenting cell activation. Cancer Immunol Immunother 57:1381-1390

19. Sheikh NA, Petrylak D, Kantoff PW et al (2013) Sipuleucel-T immune parameters correlate with survival: an analysis of the randomized phase 3 clinical trials in men with castration-resistant prostate cancer. Cancer Immunol Immunother 62:137-147

20. Sheikh N, Perdue N, Trager J, Haynes H, Guhathakurta D, Kandadi $H$ (2013) Integrated analysis of immunological profiles during manufacture of sipuleucel-T. FOCiS 2013. Abstract No. S78. http://www.focisnet.org/images/events/focis2013/Abstract\%20 Supplement_FINAL_06272013.pdf. Accessed 27 Oct 2014 
21. Fong L, Weinberg V, Chan S et al (2012) Neoadjuvant sipuleucel-T in localised prostate cancer: effects on immune cells within the prostate tumour microenvironment. European Society for Medical Oncology (ESMO) Congress, Vienna, Austria, September 28-October 2, 2012. Poster No. 939

22. McNeel D, Gardner TA, Higano CS et al (2014) A transient increase in eosinophils is associated with prolonged survival in men with metastatic castration-resistant prostate cancer who receive sipuleucel-T. Cancer Immunol Res 10:988-999

23. Jacobsen EA, Helmers RA, Lee JJ, Lee NA (2012) The expanding role of eosinophil(s) in health and disease. Blood 120:3882-3890

24. Hodi FS, O'Day SJ, McDermott DF et al (2010) Improved survival with ipilimumab in patients with metastatic melanoma. $\mathrm{N}$ Engl J Med 363:711-723

25. Madan RA, Schwaab T, Gulley JL (2012) Strategies for optimizing the clinical impact of immunotherapeutic agents such as sipuleucel-T in prostate cancer. J Natl Compr Canc Netw 10:1505-1512

26. Gulley JL, Drake CG (2011) Immunotherapy for prostate cancer: recent advances, lessons learned, and areas for further research. Clin Cancer Res 17:3884-3891

27. Huber ML, Haynes L, Parker C, Iversen P (2012) Interdisciplinary critique of sipuleucel-T as immunotherapy in castrationresistant prostate cancer. J Natl Cancer Inst 104:273-279

28. Kantoff PW, Higano CS, Small EJ, Whitmore JB, Frohlich MW, Schellhammer PF (2012) On behalf of the IMPACT authors. RE: interdisciplinary critique of sipuleucel-t as immunotherapy in castration-resistant prostate cancer. J Natl Cancer Inst 104(14):1107-1109

29. Drake CG (2012) RE: Interdisciplinary Critique of Sipuleucel$\mathrm{T}$ as Immunotherapy in Castration-Resistant Prostate Cancer. J Natl Cancer Inst 104(18):1422
30. Gulley JL, Leitman SF, Dahut W, Schlom J (2012) RE: interdisciplinary critique of sipuleucel- $\mathrm{T}$ as immunotherapy in castration-resistant prostate cancer. J Natl Cancer Inst 104(14):1106

31. Sheikh NA, Small EJ, Quinn DI et al (2012) Sipuleucel-T product characterization across different disease states of prostate cancer. J Clin Oncol 30(Suppl 5). Abstr. No. 42

32. Schellhammer PF, Chodak G, Whitmore JB, Sims R, Frohlich MW, Kantoff PW (2013) Lower baseline prostate-specific antigen is associated with a greater overall survival benefit from sipuleucel-T in the Immunotherapy for Prostate Adenocarcinoma Treatment (IMPACT) trial. Urology 81:1297-1302

33. Small EJ, Higano CS, Kantoff PW et al (2014) Time to diseaserelated pain and first opioid use in patients with metastatic castration-resistant prostate cancer treated with sipuleucel-T. Prostate Cancer Prostatic Dis 17:259-264

34. Schlom J (2012) Therapeutic cancer vaccines: current status and moving forward. J Natl Cancer Inst 104:599-613

35. Petrylak DP, Dawson NA, Gardner T et al (2010) Persistence of immunotherapy survival effects of sipuleucel-T and relationship to postrandomization docetaxel use in phase III studies. J Clin Oncol 28(Suppl 15). Abstr. No. 4551

36. Small EJ, Lance R, Gardner TA et al (2013) A randomized phase II, open-label study of sipuleucel-T with concurrent or sequential abiraterone acetate (AA) in metastatic castrate-resistant prostate cancer (mCRPC). J Clin Oncol 31(Suppl 6). Abstr. No. 114

37. Antonarakis ES, Kibel AS, Adams G, et al. (2013) A randomized phase II study evaluating the optimal sequencing of sipuleucel-T and androgen deprivation therapy (ADT) in biochemically recurrent prostate cancer (BRPC): Immune results. J Clin Oncol 31, 2013 (suppl; abstr 5016) 